\title{
Development of automated online gel permeation chromatography-gas chromatograph mass spectrometry for measuring multiresidual pesticides in agricultural products
}

\author{
Li-Bin Liu ${ }^{a}$, Yuki Hashi ${ }^{\mathrm{b}, *}$, Ya-Ping Qin ${ }^{\mathrm{b}}$, Hai-Xia Zhou ${ }^{\mathrm{b}}$, Jin-Ming Lin ${ }^{\mathrm{a}, *}$ \\ a State Key Laboratory of Environmental Chemistry and Ecotoxicology, Research Center for Eco-Environmental Sciences, \\ Chinese Academy of Sciences, P.O. Box 2871, Beijing 100085, China \\ b Shimadzu (Hong Kong) Limited, Beijing Office, Analytical Applications Center 14F No.16, Chao Yang Men Wai Street, \\ Beijing China, Life Tower Chao Yang District, Beijing 100020, China \\ Received 30 September 2005; accepted 22 July 2006 \\ Available online 23 August 2006
}

\begin{abstract}
An automated online gel permeation chromatography-gas chromatograph mass spectrometer (GPC-GC/MS) was developed for the rapid determination of residual pesticides in agricultural products. Pesticides were extracted from homogenized food samples with acetonitrile and decontaminated via the matrix solid-phase dispersion (MSPD) technique, using a primary secondary amine as sorbent prior to GPC-GC/MS analysis. A slightly modified preparation method and automated GPC step proved useful in minimizing matrix interference. To evaluate the performance of the system, 97 target pesticides were spiked at a concentration of $0.1 \mathrm{mg} / \mathrm{kg}$ into a range of food types, including potato, cabbage, carrot, apple, orange, cucumber, and rice. A low flow rate of $0.1 \mathrm{~mL} / \mathrm{min}$ in GPC resulted in a 40 -fold reduction in solvent consumption compared with conventional GPC column applications. The combination of MSPD technique and GPC-GC/MS for the analysis of the 97 pesticides can be accomplished within $90 \mathrm{~min}$. Most pesticides were recovered in the range of 70-120\%, with relative standard deviation generally less than $10 \%$. The results demonstrate that the method can be successfully applied with acceptable recoveries to a broad range of target pesticides within a diverse range of food types.
\end{abstract}

(C) 2006 Elsevier B.V. All rights reserved.

Keywords: Residual pesticides; Agricultural products; GPC-GC/MS

\section{Introduction}

In recent years, with increasing public awareness of food safety, to develop some rapidly and accurately analytical methods are required to be developed for the determination of residual pesticides in agricultural products. Generally, the complex matrix of agricultural products adversely affects analysis precision, and it is necessary to remove the matrix interference by sample pre-treatment, such as extraction and clean-up steps $[1,2]$. In order to improve the quantitative analysis, many efforts have been made to develop extraction methods and clean-up protocols [3]. However, the off-line sample preparation often suffers from time consuming, high cost and poor reproducibility.

\footnotetext{
* Corresponding author. Tel.: +86 10 62841953; fax: +86 1062841953 .

E-mail address: jmlin@mail.tsinghua.edu.cn (J.-M. Lin).
}

Therefore, an automatic sample pre-treatment is desirable and popularly studied. As one of the powerful analytical methods, gel permeation chromatography (GPC) is a recently developed and popular post-extraction cleanup method. GPC is highly effective in removing high molecular-weight interferences, such as lipids, proteins and pigments prior to analysis by gas chromatography (GC), gas chromatograph mass spectrometry (GC/MS), high-performance liquid chromatograph (HPLC), and liquid chromatograph mass spectrometry (LC/MS). The use of GPC greatly reduces the downtime of instrument, extends column life and increases the analytical precision and accuracy [4-6]. In addition, GPC has indicated the potential for automated analysis with LC or GC; several groups have successfully introduced an automated GPC clean-up technique for the determination of pesticides [7-9]. However, considering the entire analytical process, much effort is still required to reduce analysis time and cost, such as reducing the consumption of solvent. Recently, the use of 
an online gel permeation chromatography-gas chromatograph mass spectrometry (GPC-GC/MS) for the analysis of residual pesticides has been simply introduced by Hashi et al. [10].

In the present study, on the basis of our previous work [10], an automated online GPC-GC/MS for measuring residual pesticides in agricultural products is proposed. This system can determine 97 pesticides in $90 \mathrm{~min}$. A diverse range of pesticides, mainly including organophosphorus, organochlorine, organonitrogen, carbamate, and thiocarbamate substances were selected as test targets. The precision and accuracy of the method were validated by seven different agricultural products spiked with $0.1 \mathrm{mg} / \mathrm{kg}$ of residue. The matrix solid-phase dispersion (MSPD) technique with slight modification was employed for the cleanup of samples. This technique is suitable for the clean-up of homogeneously dispersed samples and can be applied to GC or GC/MS analysis [11-19]. After extraction, the samples were injected into the automated online GPC-GC/MS system. The combination of the MSPD technique and online GPC-GC/MS system enables us to accomplish a high throughput of pesticide analysis at low cost and satisfactory recovery.

\section{Experimental}

\subsection{Chemicals and solvents}

All chemicals used were of analytical reagent or chromatographic grade. Acetone, acetonitrile, and cyclohexane were obtained from Tedia Company (Fairfield, OH, USA). The HPLC grade-water was obtained by purification of de-ionized water through a Milli-Q system (Bedford, USA) with a $0.22-\mu \mathrm{m}$ fiber filter.

Analytical grade sodium chloride was obtained from Beijing Chemical Factory (Beijing, China). Anhydrous magnesium sulfate was obtained from Kanto Chemical (Tokyo, Japan). Primary secondary amine (PSA) sorbent [Bond Elut PSA] was obtained from Varian (Harbor, USA).

Fluvalinate and chinomethionate, used as markers, were both of analytical standard and obtained from Sigma-Aldrich (USA).

Two groups of pesticides stock standard solutions (for pesticide residue analysis) were obtained from Kanto Chemical
(Tokyo, Japan). One group contained 50 pesticides and another 47 pesticides, with both being dissolved in acetone. The concentrations of the two group stock standard solutions were $10 \mu \mathrm{g} / \mathrm{mL}$, and they were stored at $-25^{\circ} \mathrm{C}$. Prior to each experiment, the solutions containing 97 pesticides for analysis were prepared by mixing the two group stock standard solutions and diluting by acetone into the desired concentration.

\subsection{Apparatus}

Fig. 1 shows a schematic diagram of the gel permeation chromatography-gas chromatograph mass spectrometry system. The GPC consists of two LC-10ADvp pumps, a SIL10ADvp auto-sampler, a Shodex CLNpak EV-200AC column ( $2 \mathrm{~mm}$ i.d. $\times 150 \mathrm{~mm}$ ) and CTO-10ASvp column oven, a SPD10Avp UV detector, two FCV-12AH flow channel selection valves (RV.A, RV.B) and a SCL-10Avp system controller. GC/MS machine is a Shimadzu GC/MS-QP2010 instrumentation equipped with a PTV-2010 large-volume injection device. GC/MS data analysis was triggered by a contact closure start signal from the HPLC controller. Data acquisition was performed using a C-R8A plus data processor. All these parts are the products of Shimadzu (Kyoto, Japan), except the Shodex CLNpak EV-200AC column (Shoko Co., Tokyo, Japan). Acetone/cyclohexane mixing solvent $(3 / 7, \mathrm{v} / \mathrm{v})$ was used as the mobile phase of GPC, and the flow rate was set at $0.1 \mathrm{~mL} / \mathrm{min}$. The mobile phase was degassed using DGU-14A degasser (Shimadzu), and the GPC column was kept at $40{ }^{\circ} \mathrm{C}$ in the column oven.

\subsection{Sample preparation}

All samples were purchased at local markets in Beijing. Samples were extensively crushed to achieve good sample homogeneity. After crushing, in the case of rice, an extra step of filtering through a sifter $(0.45 \mathrm{~mm}$ aperture $)$ was required. Once homogenized, samples were stored at $-25^{\circ} \mathrm{C}$ until GPC-GC/ MS analysis.

To prepare each sample, $10 \mathrm{~g}$ of a previously homogenized food material was transferred into a suitable glass vessel (for rice,

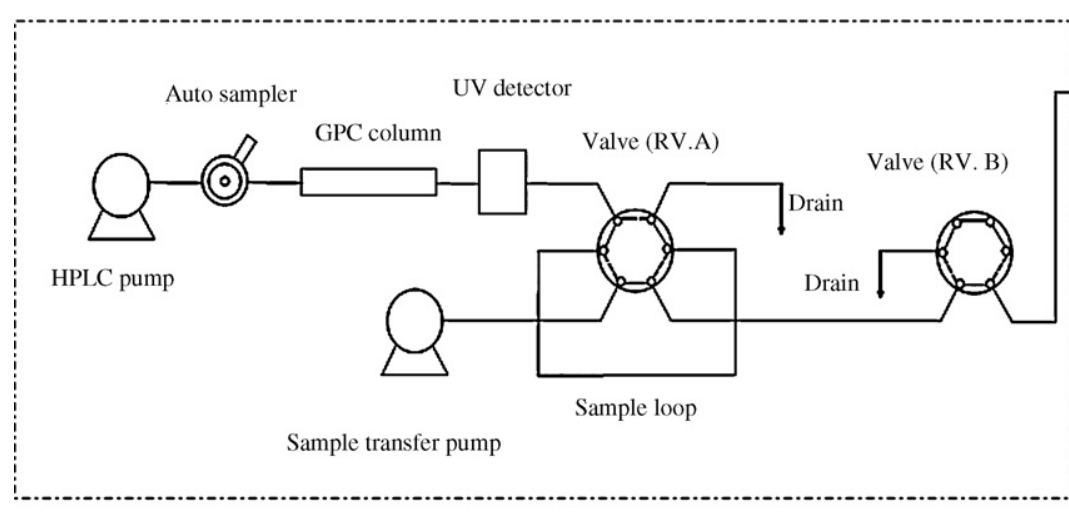

GPC

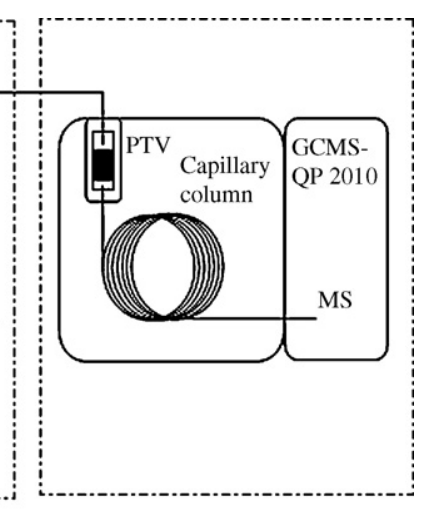

GC/MS

Fig. 1. Schematic flow diagram of the GPC-GC/MS system. 
a further $10 \mathrm{~mL}$ deionized water was added). Then, $10 \mathrm{~mL}$ acetonitrile was added to each sample using an adjustable-volume solvent dispenser. The glass vessels were capped before vortex mixing for $1 \mathrm{~min}$ at maximum speed. Once the initial sample mixing was completed, $1 \mathrm{~g} \mathrm{NaCl}$ and $4 \mathrm{~g}$ anhydrous $\mathrm{MgSO}_{4}$ were added and mixed immediately on a Vortex mixer for $1 \mathrm{~min}$. It is important to note that this step must be taken immediately after the initial mixing step to prevent the formation of $\mathrm{MgSO}_{4}$ conglomerates. To separate phases, samples were centrifuged for $10 \mathrm{~min}$ at $1570 \times \mathrm{g}$. Using an adjustable repeating pipette, $1.0 \mathrm{~mL}$ aliquot of upper acetonitrile layer was transferred into a $1.5 \mathrm{~mL}$ flip-top microcentrifuge vial containing $150 \mathrm{mg}$ anhydrous $\mathrm{MgSO}_{4}$ and $50 \mathrm{mg}$ PSA sorbent. The vial was tightly capped and shaken on a vortex mixer for $1 \mathrm{~min}$ before extraction. Then the mixed extraction solution was centrifuged for $5 \mathrm{~min}$ to separate solids from solution. The solution was then transferred into an autosampler for GPC-GC/MS analysis. For spiked samples, standard pesticides were spiked into the samples before adding acetonitrile for extraction. The other steps are the same as those described above.

\subsection{Procedure}

Before the GC/MS determination, the GPC procedure was carried out on the GPC-GC/MS system. As shown in Fig. 1,

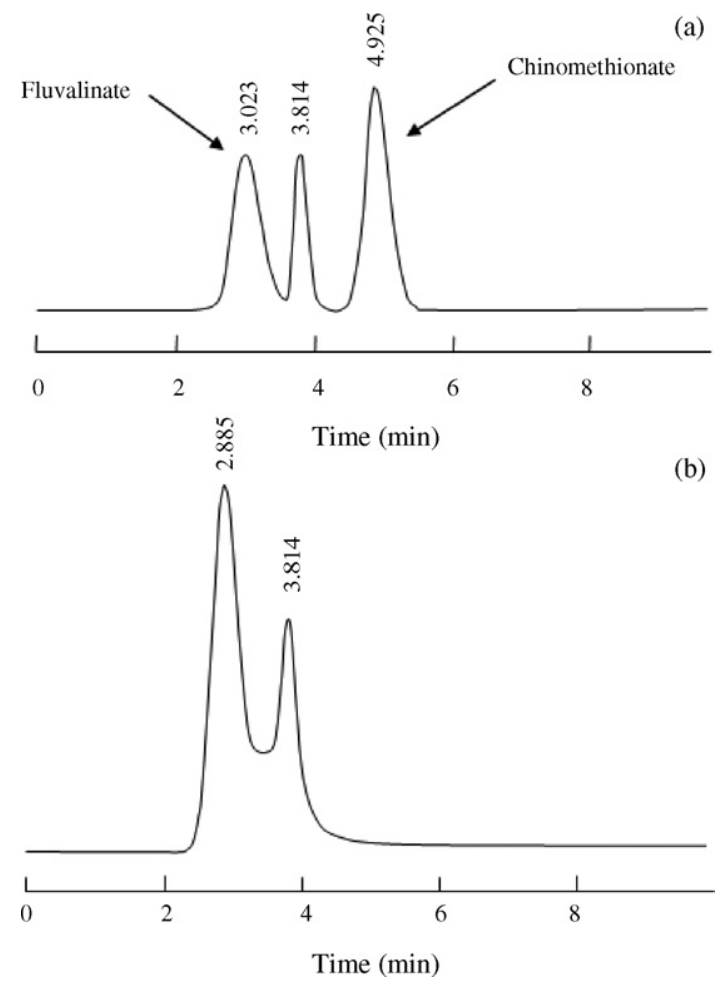

Fig. 2. GPC chromatograms obtained at a UV wavelength of $210 \mathrm{~nm}$. (a) GPC chromatogram of two markers, fluvalinate $(\mathrm{MW}=502.9)$ as the upper molecular weight marker and chinomethionate $(\mathrm{MW}=234.3)$ as the lower molecular marker at $1000 \mathrm{ng} / \mathrm{mL}$. (b) GPC chromatogram of rice sample spiked with a mixture of 97 standard pesticides at $2350.1 \mathrm{mg} / \mathrm{kg}$. Acetone/cyclohexane mixed solvent $(3 / 7, \mathrm{v} / \mathrm{v})$ was used as the mobile phase of GPC and the flow rate was set at $0.1 \mathrm{~mL} / \mathrm{min}$. The GPC column was kept at $40^{\circ} \mathrm{C}$ in the column oven.
Table 1

Retention times and quantification ions for all analyzed pesticides

\begin{tabular}{|c|c|c|c|}
\hline & Pesticide & $\begin{array}{l}\text { Retention } \\
\text { time (min) }\end{array}$ & $\begin{array}{l}\text { Quantification } \\
\text { ion }(m / z)\end{array}$ \\
\hline 1 & Methamidophos & 10.333 & 94 \\
\hline 2 & DDVP & 10.507 & 109 \\
\hline 3 & EPTC & 11.968 & 128 \\
\hline 4 & Butylate & 12.974 & 156 \\
\hline 5 & Acephate & 13.031 & 136 \\
\hline 6 & Isoprocarb & 14.497 & 121 \\
\hline 7 & BPMC & 15.474 & 121 \\
\hline 8 & Ethoprophos & 15.880 & 158 \\
\hline 9 & Chlorpropham & 16.235 & 213 \\
\hline 10 & Bendiocarb & 16.404 & 151 \\
\hline 11 & Cadusafos & 16.606 & 158 \\
\hline 12 & $\alpha-\mathrm{BHC}$ & 16.710 & 219 \\
\hline 13 & Thiometon & 16.806 & 88 \\
\hline 14 & $\beta-\mathrm{BHC}$ & 17.401 & 219 \\
\hline 15 & Dimethipin & 17.442 & 118 \\
\hline 16 & $\gamma$-BHC & 17.579 & 219 \\
\hline 17 & Terbufos & 17.786 & 231 \\
\hline 18 & Diazinon & 18.035 & 304 \\
\hline 19 & $\delta-\mathrm{BHC}$ & 18.149 & 219 \\
\hline 20 & Etrimfos & 18.326 & 292 \\
\hline 21 & Tefluthrin & 18.439 & 177 \\
\hline 22 & Ethiofencarb & 18.475 & 107 \\
\hline 23 & Pirimicarb & 18.590 & 166 \\
\hline 24 & Benfuresate & 18.955 & 121 \\
\hline 25 & Methyl-Parathion & 19.238 & 263 \\
\hline 26 & Tolclofos-methyl & 19.381 & 265 \\
\hline 27 & NAC & 19.416 & 144 \\
\hline 28 & Pirimiphos-methyl & 19.756 & 305 \\
\hline 29 & MEP & 19.860 & 277 \\
\hline 30 & Methiocarb & 19.959 & 168 \\
\hline 31 & Dichlofluanid & 20.019 & 224 \\
\hline 32 & Esprocarb & 20.080 & 222 \\
\hline 33 & Malathion & 20.165 & 173 \\
\hline 34 & Metolachlor & 20.226 & 162 \\
\hline 35 & Chlorpyrifos & 20.298 & 197 \\
\hline 36 & Thiobencarb & 20.382 & 100 \\
\hline 37 & (Z)-Dimethylvinphos & 20.429 & 295 \\
\hline 38 & Diethofencarb & 20.456 & 267 \\
\hline 39 & Parathion & 20.605 & 291 \\
\hline 40 & Isofenphos Oxon & 20.678 & 229 \\
\hline 41 & DCBP & 20.832 & 139 \\
\hline 42 & Fosthiazate & 20.886 & 195 \\
\hline 43 & Pendimethalin & 21.096 & 252 \\
\hline 44 & $\alpha-\mathrm{CVP}$ & 21.244 & 267 \\
\hline 45 & Isophenphos & 21.353 & 213 \\
\hline 46 & Captan & 21.401 & 117 \\
\hline 47 & PAP & 21.562 & 274 \\
\hline 48 & Quinalphos & 21.678 & 146 \\
\hline 49 & Tricyclazole & 21.747 & 189 \\
\hline 50 & Triadimenol & 21.815 & 112 \\
\hline 51 & Chinomethionat & 21.901 & 234 \\
\hline 52 & Pyrifenox & 21.951 & 262 \\
\hline 53 & Paclobutrazol & 22.087 & 236 \\
\hline 54 & $\beta-C V P$ & 22.466 & 267 \\
\hline 55 & Flutolanil & 22.492 & 173 \\
\hline 56 & Prothiofos & 22.509 & 309 \\
\hline 57 & Pretilachlor & 22.571 & 238 \\
\hline 58 & $p, p$ '-DDE & 22.662 & 318 \\
\hline 59 & Myclobutanil & 22.800 & 179 \\
\hline 60 & Flusilazole & 22.890 & 233 \\
\hline 61 & Cyprocpnazole & 23.197 & 222 \\
\hline 62 & Chlorobenzilate & 23.459 & 251 \\
\hline 63 & MPP & 23.520 & 278 \\
\hline
\end{tabular}


Table 1 (Continued)

\begin{tabular}{|c|c|c|c|}
\hline & Pesticide & $\begin{array}{l}\text { Retention } \\
\text { time (min) }\end{array}$ & $\begin{array}{l}\text { Quantification } \\
\text { ion }(m / z)\end{array}$ \\
\hline 64 & Fensulfothion & 23.565 & 293 \\
\hline 65 & $p, p '-\mathrm{DDD}$ & 23.676 & 235 \\
\hline 66 & Mepronil & 24.064 & 119 \\
\hline 67 & EDDP & 24.358 & 310 \\
\hline 68 & Propiconazole & 24.418 & 259 \\
\hline 69 & Lenacil & 24.486 & 153 \\
\hline 70 & Thenylchlor & 24.750 & 127 \\
\hline 71 & Tebuconazole & 24.888 & 250 \\
\hline 72 & Difolatan & 24.985 & 107 \\
\hline 73 & Acetamiprid & 25.472 & 152 \\
\hline 74 & Iprodione & 25.532 & 314 \\
\hline 75 & EPN & 25.696 & 157 \\
\hline 76 & Tebufenpyrad & 26.095 & 318 \\
\hline 77 & Phosalone & 26.462 & 182 \\
\hline 78 & Pyriproxyfen & 26.693 & 136 \\
\hline 79 & Mefenacet & 26.750 & 192 \\
\hline 80 & Cyhalothrin & 26.974 & 181 \\
\hline 81 & Fenarimol & 27.052 & 251 \\
\hline 82 & Acrinathrin & 27.254 & 181 \\
\hline 83 & Pyraclofos & 27.486 & 360 \\
\hline 84 & Bitertanol & 27.804 & 170 \\
\hline 85 & Permethrin & 27.909 & 183 \\
\hline 86 & Pyridaben & 28.109 & 147 \\
\hline 87 & Cyfluthrin & 28.800 & 163 \\
\hline 88 & Halfenprox & 29.062 & 263 \\
\hline 89 & Cypermethrin & 29.133 & 163 \\
\hline 90 & Flucythrinate & 29.471 & 199 \\
\hline 91 & Silafluofen & 29.574 & 286 \\
\hline 92 & Pyrimidufen & 29.872 & 184 \\
\hline 93 & Fenvalerate & 30.073 & 225 \\
\hline 94 & Fluvalinate & 30.343 & 250 \\
\hline 95 & Difenoconazole & 30.673 & 323 \\
\hline 96 & Deltamethrin & 30.988 & 253 \\
\hline 97 & Imibenconazole & 32.001 & 125 \\
\hline
\end{tabular}

acetonitrile extracts of samples were injected onto the GPC column. Acetone/cyclohexane mixed solvent (3/7, v/v) was used as the sample delivery solvent. Sample clean-up and transfer were achieved by changing the flow line on the flow channel selection valves (RV.A, RV.B). The LC-10ADvp pump transferred the target pesticides to the sample loop and then delivered them to the PTV injector. In this system, a semi-micro GPC column was employed to reduce mobile phase consumption and lower operating costs. In this experiment, the injection volume was $10 \mu \mathrm{L}$ and the volume of the sample loop was set to $200 \mu \mathrm{L}$.

For the GC/MS determination, the temperature of the PTV injector was set at $120^{\circ} \mathrm{C}$ for the initial $5 \mathrm{~min}$ of sampling time, and then increased to $250^{\circ} \mathrm{C}$ at $100^{\circ} \mathrm{C} / \mathrm{min}$. An RTX-5 ms column ((5\% phenyl) methylpolysiloxane; $0.25 \mathrm{~mm}$ i.d. $\times 30 \mathrm{~m}$ with a film thickness of $0.25 \mu \mathrm{m}$; Restek Corporation, Bellefonte, PA, USA) was used for the separation of the pesticides. The temperature of the capillary column was set at $82^{\circ} \mathrm{C}$ for the initial $5 \mathrm{~min}$ and increased to $300^{\circ} \mathrm{C}$ at $8^{\circ} \mathrm{C} / \mathrm{min}$. Helium was used as the carrier gas. The quadrupole mass spectrometer was operated in the electron impact ion (EI) mode with a source temperature of $230^{\circ} \mathrm{C}$ and electron energy of $70 \mathrm{eV}$. Chromatograms
Table 2

Regression data for representative pesticides

\begin{tabular}{|c|c|c|c|c|}
\hline \multirow[t]{2}{*}{ Pesticide } & \multicolumn{3}{|c|}{$y=a x^{2}+b x+c$} & \multirow[t]{2}{*}{$r$} \\
\hline & $a$ & $b$ & $c$ & \\
\hline EPTC & 4.3355 & 7593.205 & 60394.5 & 0.99 \\
\hline Butylate & 2.731533 & 3955.23 & 47570.17 & 0.9997 \\
\hline$\alpha-\mathrm{BHC}$ & 1.55405 & 2477.382 & 13524.25 & 0.99 \\
\hline 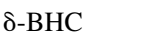 & 1.156636 & 1558.815 & 10873.18 & 0.999 \\
\hline NAC & 13.95821 & 11939.0 & -65252.46 & 0.99 \\
\hline Diethofencarb & 2.132611 & 933.7883 & 10793.56 & 0.99 \\
\hline MPP & 11.30101 & 4272.468 & -29395.44 & 0.998 \\
\hline Chlorpyrifos & 1.481919 & 2362.142 & -7932.403 & 0.99 \\
\hline Tebufenpyrad & 3.301733 & 2073.86 & 15727.67 & 0.999 \\
\hline Pyridaben & 22.14579 & 8767.302 & 76838.93 & 0.99 \\
\hline
\end{tabular}

$y$ and $x$ are the peak area and concentration $(\mathrm{ng} / \mathrm{ml})$ of the pesticides, respectively. Correlation coefficients are expressed as $r$.

were acquired in 'scan' mode, scanning from $\mathrm{m} / \mathrm{z} 86$ to $\mathrm{m} / \mathrm{z}$ 500 .

\section{Results and discussion}

\subsection{GPC conditions}

In optimizing the transfer of solvent between the GPC and GC/MS systems, the GPC mobile phase flow rate was reduced compared to that in conventional GPC. In this application, a flow rate of $0.1 \mathrm{~mL} / \mathrm{min}$ was used with a $2 \mathrm{~mm}$ i.d. GPC column; this resulted in a 40-fold reduction in solvent consumption compared to conventional GPC column applications. To investigate the fraction time of the pesticides, two marker molecules were selected. Fluvalinate (MW $=502.9$ ) was used as the upper molecular weight marker and Chinomethionate $(\mathrm{MW}=234.3)$ was used as the lower molecular weight marker, corresponding to a retention time of between 3.023 and $4.925 \mathrm{~min}$ (the GPC chromatograms of the two marker molecules and the rice sample spiked with 97 pesticides standard are shown in Fig. 2). In this study, GPC eluent from 2.9 to 4.9 min was fractionated by the sample loop.

Table 3

Estimated limits of detection (LOD) and quantitation (LOQ) calculated as the concentrations that produced a signal equal to 3-times and 10-times the background noise level, respectively, in GPC-GC/MS

\begin{tabular}{|c|c|c|c|c|c|c|}
\hline \multirow[t]{2}{*}{ Pesticide } & \multicolumn{3}{|c|}{$\operatorname{LOD}(\mu \mathrm{g} / \mathrm{kg})$} & \multicolumn{3}{|c|}{ LOQ $(\mu \mathrm{g} / \mathrm{kg})$} \\
\hline & Potato & Apple & Rice & Potato & Apple & Rice \\
\hline EPTC & 9 & 18 & 13 & 31 & 59 & 44 \\
\hline Butylate & 38 & 79 & 30 & 127 & 262 & 101 \\
\hline$\alpha-\mathrm{BHC}$ & 15 & 14 & 16 & 51 & 48 & 52 \\
\hline$\delta$-BHC & 17 & 18 & 26 & 56 & 57 & 87 \\
\hline NAC & 3 & 21 & 18 & 11 & 69 & 60 \\
\hline Diethofencarb & 26 & 24 & 15 & 85 & 75 & 49 \\
\hline MPP & 29 & 8 & 8 & 97 & 27 & 28 \\
\hline Chlorpyrifos & 27 & 22 & 18 & 88 & 72 & 61 \\
\hline Tebufenpyrad & 10 & 16 & 14 & 36 & 53 & 47 \\
\hline Pyridaben & 7 & 14 & 17 & 23 & 46 & 55 \\
\hline
\end{tabular}




\subsection{Analysis time}

By combining automated GPC clean-up with GC/MS analysis, the total analysis time for 97 target pesticides was only $50 \mathrm{~min}$. As $40 \mathrm{~min}$ is sufficient for sample preparation, the combination of the MSPD technique and GPC-GC/MS instrument enables us to complete the analysis of 97 residual pesticides within $90 \mathrm{~min}$ per sample. The retention times and ions used for quantification of the pesticides are shown in Table 1.
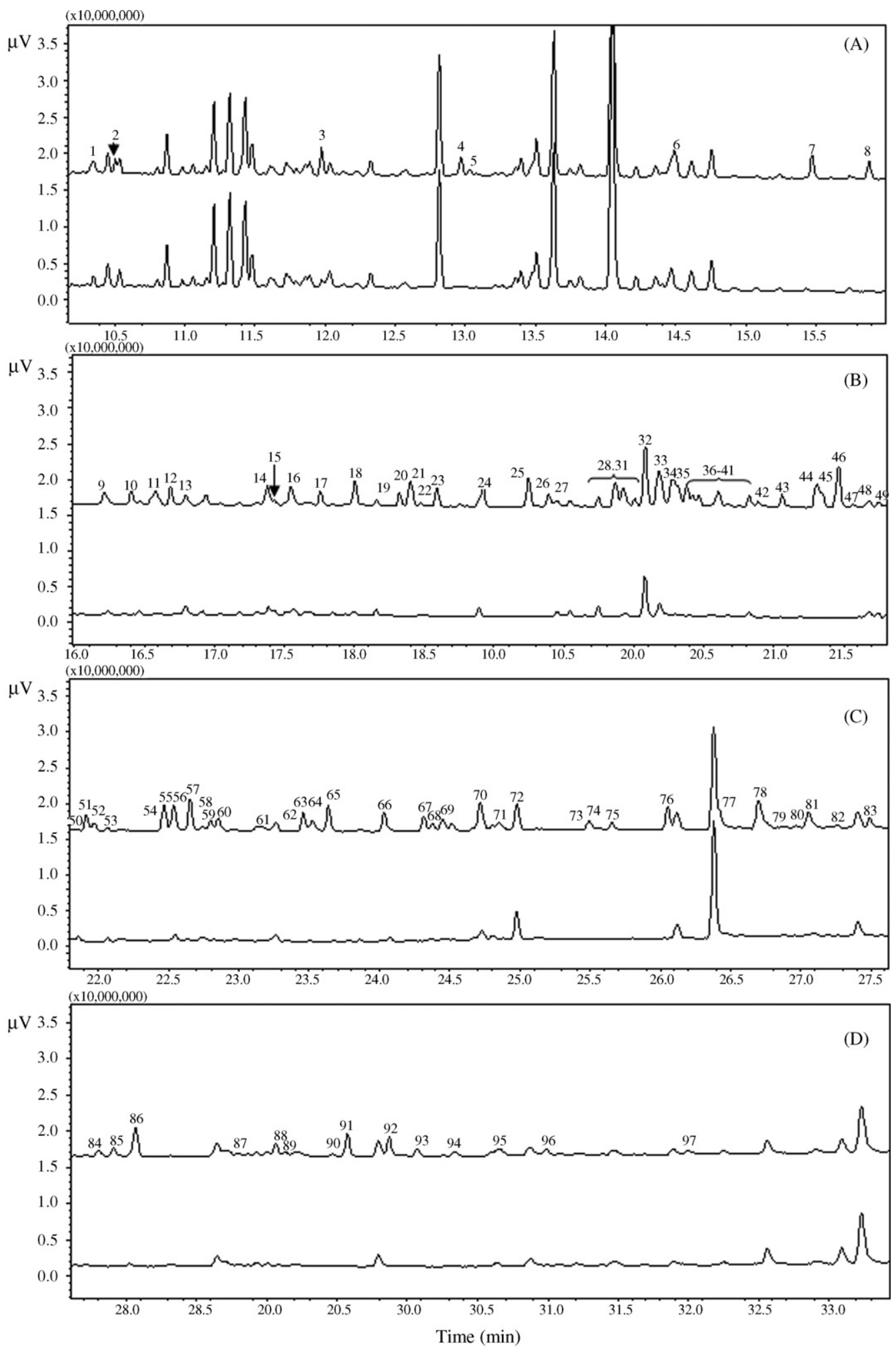

Fig. 3. Total ion chromatograms (TIC) of potato spiked with 97 pesticides at $0.1 \mathrm{mg} / \mathrm{kg}$ (upper chromatogram) and unspiked potato (lower chromatogram). Multiple chromatograms (A, B, C and D) are used to show all the pesticides. The order of peaks corresponds to the order of pesticides listed in Table 1. 
Table 4

Comparison of recovery (\%) achieved by GPC-GC/MS and GC/MS

\begin{tabular}{|c|c|c|c|c|c|c|c|c|c|c|c|c|c|c|c|c|c|c|c|c|c|c|}
\hline & \multirow[t]{3}{*}{ Pesticide } & \multirow{2}{*}{\multicolumn{3}{|c|}{$\begin{array}{l}\text { Potato } \\
\text { GPC-GC/MS } G C / M S\end{array}$}} & \multicolumn{3}{|c|}{ Cabbage } & \multicolumn{3}{|l|}{ Carrot } & \multicolumn{3}{|c|}{ Cucumber } & \multicolumn{3}{|l|}{ Apple } & \multicolumn{3}{|l|}{ Orange } & \multicolumn{3}{|l|}{ Rice } \\
\hline & & & & & \multicolumn{2}{|c|}{$\overline{\mathrm{GPC}-\mathrm{GC} / \mathrm{MS}}$} & \multirow{2}{*}{$\begin{array}{l}\text { GC/MS } \\
\text { Mean } \\
(\%)\end{array}$} & \multicolumn{2}{|c|}{$\overline{\mathrm{GPC}-\mathrm{GC} / \mathrm{MS}}$} & \multirow{2}{*}{$\begin{array}{l}\text { GC/MS } \\
\text { Mean } \\
(\%)\end{array}$} & \multicolumn{2}{|c|}{ GPC-GC/MS } & & $\overline{\text { GPC-C }}$ & C/MS & $\overline{\mathrm{GC} / \mathrm{MS}}$ & $\overline{\text { GPC-C }}$ & C/MS & $\overline{\mathrm{GC} / \mathrm{MS}}$ & $\overline{\text { GPC-C }}$ & C/MS & $\overline{\mathrm{GC} / \mathrm{MS}}$ \\
\hline & & $\begin{array}{l}\text { Mean } \\
(\%)\end{array}$ & $\begin{array}{l}\text { R.S.D. } \\
(\%)\end{array}$ & $\begin{array}{l}\text { Mean } \\
(\%)\end{array}$ & $\begin{array}{l}\text { Mean } \\
(\%)\end{array}$ & $\begin{array}{l}\text { R.S.D. } \\
(\%)\end{array}$ & & $\begin{array}{l}\text { Mean } \\
(\%)\end{array}$ & $\begin{array}{l}\text { R.S.D. } \\
(\%)\end{array}$ & & $\begin{array}{l}\text { Mean } \\
(\%)\end{array}$ & $\begin{array}{l}\text { R.S.D. } \\
(\%)\end{array}$ & $\begin{array}{l}\text { Mean } \\
(\%)\end{array}$ & $\begin{array}{l}\text { Mean } \\
(\%)\end{array}$ & $\begin{array}{l}\text { R.S.D. } \\
(\%)\end{array}$ & $\begin{array}{l}\text { Mean } \\
(\%)\end{array}$ & $\begin{array}{l}\text { Mean } \\
(\%)\end{array}$ & $\begin{array}{l}\text { R.S.D. } \\
(\%)\end{array}$ & $\begin{array}{l}\text { Mean } \\
(\%)\end{array}$ & $\begin{array}{l}\text { Mean } \\
(\%)\end{array}$ & $\begin{array}{l}\text { R.S.D. } \\
(\%)\end{array}$ & $\begin{array}{l}\text { Mean } \\
(\%)\end{array}$ \\
\hline 1 & Acephate & 73 & 0.3 & 194 & 74 & 1.1 & 205 & 70 & 1.3 & 220 & 59 & 1.3 & 75 & 57 & 3.5 & 116 & 55 & 2.9 & 108 & 60 & 5.7 & 108 \\
\hline 2 & Acetamiprid & 71 & 10.7 & 89 & 91 & 2.8 & 100 & 99 & 4.4 & 108 & 88 & 4.1 & 106 & 86 & 9.8 & 88 & 99 & 11.6 & 101 & 92 & 3.6 & 198 \\
\hline 3 & Acrinathrin & 115 & 5.7 & 85 & 135 & 5.4 & 113 & 188 & 2.8 & 88 & 110 & 4.5 & 104 & 134 & 1.4 & 116 & 121 & 2.3 & 120 & 111 & 7.6 & 114 \\
\hline 4 & Bendiocarb & 86 & 0.3 & 71 & 87 & 2.1 & 94 & 92 & 3.0 & 75 & 100 & 1.3 & 97 & 87 & 3.5 & 107 & 106 & 3.5 & 165 & 101 & 1.9 & 111 \\
\hline 5 & Benfuresate & 94 & 1.0 & 75 & 94 & 0.9 & 77 & 102 & 0.8 & 77 & 108 & 1.3 & 71 & 96 & 1.8 & 78 & 111 & 2.4 & 114 & 97 & 3.2 & 87 \\
\hline 6 & $\alpha$-BHC & 97 & 1.5 & 76 & 97 & 1.0 & 79 & 102 & 2.4 & 79 & 113 & 3.8 & 73 & 104 & 2.4 & 56 & 114 & 5.0 & 107 & 118 & 2.5 & 69 \\
\hline 7 & $\beta$-BHC & 107 & 0.3 & 80 & 106 & 3.2 & 83 & 112 & 4.1 & 83 & 103 & 1.1 & 90 & 120 & 1.7 & 99 & 137 & 3.5 & 113 & 104 & 1.3 & 89 \\
\hline 8 & $\gamma$-BHC & 99 & 0.9 & 76 & 101 & 2.6 & 77 & 106 & 1.6 & 76 & 112 & 2.4 & 84 & 108 & 2.0 & 63 & 116 & 4.5 & 114 & 105 & 2.9 & 72 \\
\hline 9 & $\delta$-BHC & 105 & 1.1 & 66 & 106 & 2.7 & 82 & 108 & 0.7 & 75 & 110 & 1.2 & 68 & 115 & 1.2 & 53 & 111 & 4.3 & 76 & 110 & 2.0 & 56 \\
\hline 10 & Bitertanol & 125 & 2.2 & 95 & 119 & 2.4 & 91 & 100 & 7.5 & 114 & 118 & 1.1 & 189 & 109 & 1.3 & 115 & 111 & 7.8 & 130 & 117 & 2.5 & 199 \\
\hline 11 & BPMC & 98 & 1.7 & 79 & 97 & 1.3 & 86 & 100 & 1.4 & 74 & 104 & 1.4 & 93 & 109 & 1.3 & 79 & 119 & 1.4 & 119 & 111 & 1.9 & 83 \\
\hline 12 & Butylate & 110 & 1.2 & 68 & 111 & 3.4 & 69 & 110 & 2.7 & 69 & 100 & 1.9 & 64 & 90 & 0.5 & 57 & 112 & 4.0 & 93 & 106 & 5.5 & 60 \\
\hline 13 & Cadusafos & 104 & 0.7 & 74 & 102 & 2.3 & 77 & 108 & 2.8 & 80 & 110 & 2.1 & 92 & 118 & 3.3 & 81 & 112 & 6.7 & 97 & 116 & 1.6 & 84 \\
\hline 14 & Captan & 91 & 3.0 & 69 & 66 & 1.4 & ND & 77 & 2.4 & 51 & 110 & 5.1 & 37 & 101 & 0.4 & 61 & 145 & 6.3 & 86 & 26 & 5.6 & 25 \\
\hline 15 & Chinomethionat & 56 & 1.8 & 49 & 55 & 2.1 & 49 & 55 & 2.3 & 48 & 16 & 5.3 & 43 & 19 & 10.6 & 44 & 35 & 4.2 & 53 & 22 & 9.9 & 41 \\
\hline 16 & Chlorobenzilate & 114 & 1.5 & 86 & 111 & 3.0 & 84 & 117 & 1.8 & 92 & 115 & 5.0 & 129 & 127 & 4.3 & 116 & 115 & 3.8 & 148 & 148 & 4.6 & 143 \\
\hline 17 & Chlorpropham & 112 & 1.9 & 72 & 113 & 1.2 & 77 & 115 & 3.5 & 76 & 118 & 1.7 & 77 & 109 & 1.3 & 72 & 117 & 0.8 & 110 & 117 & 1.7 & 77 \\
\hline 18 & Chlorpyrifos & 97 & 0.8 & 70 & 95 & 3.3 & 71 & 100 & 4.0 & 73 & 113 & 0.9 & 88 & 114 & 1.3 & 82 & 111 & 5.3 & 120 & 116 & 3.2 & 98 \\
\hline 19 & $\alpha-C V P$ & 103 & 4.1 & 91 & 104 & 3.5 & 95 & 107 & 1.7 & 94 & 115 & 2.2 & 119 & 112 & 2.2 & 116 & 105 & 4.2 & 160 & 115 & 2.8 & 117 \\
\hline 20 & $\beta$-CVP & 104 & 0.5 & 91 & 100 & 2.7 & 95 & 106 & 2.2 & 94 & 116 & 2.2 & 119 & 112 & 2.2 & 119 & 105 & 4.2 & 153 & 117 & 3.6 & 130 \\
\hline 21 & Cyfluthrin & 92 & 7.2 & 67 & 101 & 2.6 & 79 & 124 & 7.0 & 75 & 101 & 0.9 & 105 & 96 & 4.9 & 110 & 115 & 7.4 & 95 & 112 & 1.3 & 116 \\
\hline 22 & Cyhalothrin & 112 & 2.5 & 86 & 121 & 2.9 & 112 & 118 & 2.5 & 110 & 115 & 3.5 & 113 & 99 & 0.5 & 107 & 154 & 1.3 & 118 & 150 & 1.4 & 132 \\
\hline 23 & Cypermethrin & 106 & 4.6 & 55 & 104 & 3.6 & 85 & 103 & 1.8 & 79 & 112 & 4.8 & 91 & 124 & 2.7 & 79 & 111 & 2.5 & 91 & 117 & 2.8 & 98 \\
\hline 24 & Cyproconazole & 106 & 4.3 & 83 & 105 & 4.3 & 84 & 117 & 4.1 & 91 & 100 & 3.3 & 125 & 113 & 1.1 & 109 & 102 & 4.8 & 139 & 155 & 4.3 & 134 \\
\hline 25 & DCBP & 104 & 6.0 & 78 & 108 & 4.7 & 82 & 120 & 1.7 & 80 & 191 & 6.9 & 88 & 181 & 0.8 & 81 & 159 & 8.1 & 114 & 197 & 7.8 & 95 \\
\hline 26 & $p, p^{\prime}$-DDD & 105 & 0.3 & 82 & 104 & 1.9 & 87 & 107 & 2.0 & 89 & 110 & 1.8 & 103 & 119 & 1.1 & 85 & 113 & 5.2 & 118 & 120 & 7.1 & 114 \\
\hline 27 & $p, p^{\prime}$-DDE & 100 & 4.0 & 82 & 97 & 1.6 & 82 & 95 & 0.8 & 81 & 113 & 0.3 & 85 & 109 & 2.6 & 80 & 120 & 5.3 & 115 & 113 & 4.8 & 87 \\
\hline 28 & DDVP & 88 & 0.8 & 72 & 88 & 1.7 & 78 & 91 & 1.3 & 73 & 98 & 2.1 & 74 & 85 & 1.1 & 67 & 98 & 4.3 & 99 & 105 & 0.8 & 64 \\
\hline 29 & Deltamethrin & 109 & 3.3 & 78 & 95 & 6.1 & 81 & 111 & 4.3 & 108 & 64 & 2.5 & 62 & 62 & 0.7 & 92 & 63 & 1.6 & 82 & 44 & 0.6 & 31 \\
\hline 30 & Diazinon & 106 & 0.7 & 76 & 104 & 3.4 & 78 & 109 & 2.2 & 79 & 121 & 0.7 & 86 & 111 & 1.4 & 83 & 119 & 3.8 & 116 & 120 & 3.2 & 86 \\
\hline 31 & Dichlofluanid & 72 & 5.6 & 63 & 62 & 2.0 & 58 & 50 & 3.9 & 61 & 37 & 0.2 & 47 & 61 & 8.4 & 58 & 56 & 7.6 & 81 & 20 & 5.5 & 44 \\
\hline 32 & Diethofencarb & 117 & 3.1 & 86 & 106 & 4.9 & 84 & 115 & 2.1 & 89 & 114 & 2.1 & 102 & 99 & 2.7 & 102 & 115 & 3.1 & 152 & 132 & 0.9 & 128 \\
\hline 33 & Difenoconazole & 123 & 4.1 & 95 & 115 & 8.9 & 87 & 137 & 8.1 & 117 & 163 & 3.0 & 143 & 113 & 1.8 & 111 & 112 & 5.0 & 142 & 112 & 4.8 & 144 \\
\hline 34 & Difolatan & 90 & 4.2 & $N D$ & 58 & 7.0 & ND & 112 & 5.3 & ND & 65 & 5.6 & 45 & 68 & 5.6 & 96 & 87 & 3.8 & 53 & 19 & 5.5 & 46 \\
\hline 35 & Dimethipin & 77 & 1.3 & 84 & 79 & 3.5 & 89 & 79 & 1.7 & 73 & 72 & 3.7 & 85 & 54 & 1.5 & 85 & 71 & 7.4 & 112 & 72 & 2.3 & 73 \\
\hline 36 & (Z)-Dimethylvinphos & 97 & 1.6 & 94 & 94 & 2.1 & 99 & 102 & 2.0 & 97 & 112 & 4.7 & 112 & 104 & 0.6 & 116 & 115 & 3.4 & 164 & 113 & 2.4 & 116 \\
\hline 37 & EDDP & 107 & 0.9 & 132 & 107 & 1.3 & 143 & 118 & 2.8 & 128 & 94 & 2.3 & 143 & 119 & 2.0 & 136 & 116 & 1.8 & 180 & 115 & 2.3 & 169 \\
\hline 38 & EPN & 110 & 0.9 & 95 & 112 & 2.9 & 97 & 138 & 4.7 & 113 & 94 & 4.8 & 158 & 116 & 1.5 & 103 & 97 & 6.3 & 113 & 112 & 4.8 & 183 \\
\hline 39 & EPTC & 99 & 1.0 & 69 & 97 & 1.5 & 69 & 101 & 1.7 & 70 & 120 & 0.2 & 60 & 111 & 1.1 & 50 & 118 & 2.9 & 84 & 116 & 1.3 & 60 \\
\hline 40 & Esprocarb & 99 & 1.3 & 77 & 98 & 1.9 & 79 & 103 & 1.4 & 82 & 117 & 0.7 & 91 & 111 & 3.0 & 85 & 117 & 1.2 & 116 & 116 & 2.3 & 94 \\
\hline 41 & Ethiofencarb & 94 & 5.1 & 105 & 92 & 2.5 & 121 & 91 & 3.3 & 98 & 72 & 7.4 & 113 & 95 & 6.4 & 115 & 88 & 6.8 & 177 & 77 & 3.7 & 112 \\
\hline 42 & Ethoprophos & 99 & 0.5 & 78 & 100 & 1.9 & 78 & 103 & 3.0 & 82 & 112 & 1.4 & 84 & 113 & 2.0 & 85 & 114 & 0.8 & 118 & 107 & 1.8 & 79 \\
\hline 43 & Etrimfos & 103 & 0.6 & 74 & 101 & 2.3 & 75 & 107 & 3.4 & 78 & 119 & 1.5 & 103 & 119 & 1.2 & 93 & 110 & 6.1 & 132 & 114 & 2.5 & 95 \\
\hline 44 & Fenarimol & 100 & 1.3 & 85 & 99 & 3.0 & 85 & 111 & 3.0 & 95 & 108 & 1.7 & 132 & 118 & 1.2 & 110 & 112 & 5.9 & 141 & 112 & 4.3 & 143 \\
\hline 45 & Fensulfothion & 99 & 1.5 & 89 & 102 & 1.8 & 97 & 119 & 1.4 & 122 & 110 & 5.7 & 136 & 101 & 1.6 & 110 & 78 & 6.2 & 110 & 111 & 6.0 & 140 \\
\hline 46 & Fenvalerate & 101 & 6.5 & 74 & 101 & 2.2 & 105 & 104 & 2.1 & 89 & 113 & 3.4 & 81 & 115 & 2.0 & 113 & 119 & 1.0 & 111 & 112 & 2.0 & 115 \\
\hline
\end{tabular}




\begin{tabular}{|c|c|c|c|c|c|c|c|c|c|c|c|c|c|c|c|c|c|c|c|c|c|c|}
\hline 47 & Flucythrinate & 114 & 2.8 & 93 & 120 & 2.8 & 111 & 120 & 3.8 & 110 & 153 & 5.8 & 116 & 110 & 4.2 & 119 & 118 & 5.1 & 129 & 117 & 6.3 & 116 \\
\hline 48 & Flusilazole & 93 & 1.2 & 80 & 92 & 2.5 & 85 & 97 & 2.1 & 87 & 116 & 5.0 & 115 & 98 & 4.7 & 107 & 117 & 1.8 & 120 & 111 & 3.3 & 117 \\
\hline 49 & Flutolanil & 113 & 2.6 & 85 & 110 & 1.8 & 88 & 110 & 5.4 & 104 & 116 & 6.4 & 120 & 106 & 0.6 & 115 & 114 & 6.8 & 139 & 157 & 1.0 & 130 \\
\hline 50 & Fluvalinate & 142 & 5.9 & 82 & 155 & 0.5 & 133 & 152 & 1.6 & 75 & 145 & 4.0 & 112 & 135 & 2.1 & 127 & 151 & 3.7 & 113 & 162 & 4.8 & 129 \\
\hline 51 & Fosthiazate & 108 & 0.7 & 127 & 105 & 3.6 & 127 & 118 & 3.9 & 129 & 103 & 3.3 & 110 & 106 & 7.3 & 147 & 105 & 5.9 & 193 & 90 & 4.2 & 92 \\
\hline 52 & Halfenprox & 94 & 1.1 & 92 & 97 & 1.6 & 99 & 103 & 3.7 & 115 & 158 & 1.0 & 120 & 106 & 1.5 & 120 & 117 & 2.0 & 130 & 120 & 2.6 & 139 \\
\hline 53 & Imibenconazole & 157 & 8.1 & 88 & 156 & 9.2 & 99 & 180 & 5.9 & 113 & 119 & 3.2 & 110 & 110 & 2.6 & 75 & 115 & 6.3 & 107 & 105 & 5.1 & 104 \\
\hline 54 & Iprodione & 103 & 0.8 & 138 & 104 & 2.6 & 156 & 112 & 1.7 & 91 & 104 & 1.1 & 111 & 120 & 0.7 & 117 & 106 & 4.3 & 97 & 119 & 1.5 & 136 \\
\hline 55 & Isofenphos Oxon & 120 & 2.8 & 118 & 117 & 2.8 & 112 & 88 & 6.0 & 110 & 102 & 3.2 & 191 & 116 & 3.8 & 167 & 105 & 4.0 & 178 & 139 & 1.4 & 195 \\
\hline 56 & Isophenphos & 116 & 1.5 & 80 & 114 & 2.7 & 83 & 120 & 1.2 & 88 & 118 & 2.5 & 118 & 115 & 0.8 & 112 & 118 & 2.1 & 135 & 144 & 1.7 & 118 \\
\hline 57 & Isoprocarb & 95 & 0.6 & 80 & 96 & 2.1 & 87 & 96 & 2.1 & 69 & 112 & 1.3 & 88 & 100 & 0.8 & 81 & 113 & 3.5 & 114 & 115 & 0.7 & 83 \\
\hline 58 & Lenacil & 109 & 0.6 & 91 & 107 & 2.1 & 92 & 119 & 2.4 & 104 & 111 & 7.4 & 124 & 112 & 2.4 & 118 & 117 & 2.9 & 134 & 168 & 2.9 & 143 \\
\hline 59 & Malathion & 97 & 0.8 & 83 & 95 & 1.3 & 91 & 104 & 2.1 & 90 & 116 & 3.1 & 113 & 110 & 1.4 & 106 & 98 & 4.3 & 139 & 119 & 0.7 & 112 \\
\hline 60 & Mefenacet & 104 & 1.3 & 93 & 108 & 1.7 & 95 & 116 & 3.4 & 107 & 96 & 3.2 & 125 & 93 & 3.2 & 115 & 96 & 7.6 & 131 & 99 & 4.7 & 144 \\
\hline 61 & MEP & 100 & 2.1 & 81 & 102 & 1.5 & 74 & 112 & 3.7 & 94 & 113 & 2.5 & 120 & 119 & 0.6 & 119 & 87 & 6.4 & 156 & 114 & 6.4 & 125 \\
\hline 62 & Mepronil & 109 & 2.0 & 85 & 110 & 2.3 & 85 & 119 & 1.6 & 93 & 112 & 0.8 & 120 & 119 & 2.2 & 116 & 116 & 2.1 & 129 & 153 & 3.6 & 134 \\
\hline 63 & Methamidophos & 68 & 1.2 & 71 & 72 & 2.0 & 84 & 71 & 0.8 & 68 & 61 & 9.3 & 109 & 57 & 5.9 & 110 & 56 & 3.6 & 111 & 48 & 5.0 & 103 \\
\hline 64 & Methiocarb & 112 & 2.1 & 90 & 114 & 3.0 & 126 & 118 & 2.1 & 80 & 115 & 1.5 & 134 & 103 & 2.2 & 113 & 115 & 2.8 & 185 & 117 & 2.2 & 109 \\
\hline 65 & Methyl-Parathion & 104 & 1.4 & 85 & 120 & 3.5 & 82 & 115 & 4.6 & 92 & 119 & 1.4 & 108 & 118 & 2.7 & 115 & 84 & 2.1 & 151 & 114 & 2.0 & 120 \\
\hline 66 & Metolachlor & 102 & 0.3 & 80 & 99 & 2.5 & 80 & 107 & 2.2 & 86 & 107 & 1.4 & 118 & 116 & 0.6 & 100 & 106 & 8.3 & 139 & 118 & 2.4 & 117 \\
\hline 67 & MPP & 63 & 1.1 & 76 & 57 & 1.8 & 81 & 56 & 1.7 & 83 & 149 & 1.3 & 95 & 108 & 2.5 & 89 & 118 & 2.2 & 112 & 97 & 5.6 & 88 \\
\hline 68 & Myclobutanil & 91 & 2.1 & 84 & 92 & 1.6 & 84 & 98 & 2.2 & 93 & 91 & 1.8 & 110 & 110 & 1.9 & 111 & 93 & 6.2 & 112 & 118 & 0.5 & 128 \\
\hline 69 & NAC & 99 & 0.9 & 78 & 106 & 2.6 & 124 & 109 & 5.2 & 78 & 113 & 1.5 & 115 & 98 & 0.6 & 120 & 110 & 6.9 & 146 & 111 & 3.7 & 111 \\
\hline 70 & Paclobutrazol & 106 & 0.1 & 84 & 102 & 2.9 & 83 & 112 & 4.1 & 92 & 106 & 6.2 & 118 & 115 & 0.9 & 110 & 108 & 3.7 & 150 & 120 & 7.8 & 81 \\
\hline 71 & PAP & 98 & 0.8 & 78 & 95 & 2.2 & 80 & 106 & 3.2 & 89 & 119 & 1.3 & 111 & 118 & 2.8 & 103 & 102 & 5.6 & 135 & 112 & 6.2 & 114 \\
\hline 72 & Parathion & 114 & 1.9 & 83 & 108 & 3.8 & 77 & 120 & 3.2 & 96 & 119 & 1.1 & 136 & 84 & 1.6 & 118 & 101 & 5.6 & 158 & 117 & 4.1 & 135 \\
\hline 73 & Pendimethalin & 110 & 0.5 & 85 & 108 & 3.0 & 79 & 120 & 2.7 & 96 & 93 & 3.5 & 129 & 119 & 1.0 & 114 & 102 & 6.5 & 154 & 117 & 2.2 & 138 \\
\hline 74 & Permethrin & 113 & 0.4 & 92 & 111 & 2.6 & 95 & 115 & 2.6 & 98 & 117 & 0.5 & 108 & 111 & 3.4 & 112 & 116 & 5.4 & 109 & 114 & 3.6 & 119 \\
\hline 75 & Phosalone & 111 & 0.5 & 87 & 116 & 2.3 & 95 & 120 & 2.6 & 103 & 105 & 4.1 & 131 & 94 & 2.0 & 115 & 146 & 4.4 & 97 & 113 & 3.0 & 151 \\
\hline 76 & Pirimicarb & 92 & 0.5 & 76 & 89 & 1.8 & 78 & 97 & 2.6 & 80 & 107 & 2.1 & 82 & 97 & 0.6 & 79 & 107 & 6.5 & 114 & 110 & 2.7 & 89 \\
\hline 77 & Pirimiphos-methyl & 103 & 0.5 & 76 & 102 & 3.0 & 82 & 108 & 1.4 & 84 & 111 & 2.2 & 103 & 118 & 1.2 & 98 & 114 & 4.8 & 131 & 118 & 2.1 & 112 \\
\hline 78 & Pretilachlor & 114 & 0.5 & 88 & 113 & 1.8 & 83 & 118 & 7.8 & 101 & 117 & 1.8 & 134 & 119 & 0.2 & 120 & 120 & 0.2 & 164 & 118 & 3.4 & 146 \\
\hline 79 & Propiconazole & 97 & 0.6 & 85 & 98 & 4.2 & 86 & 105 & 2.9 & 92 & 94 & 1.2 & 143 & 94 & 2.5 & 113 & 94 & 3.9 & 115 & 110 & 6.2 & 136 \\
\hline 80 & Prothiofos & 103 & 0.8 & 80 & 101 & 1.2 & 83 & 86 & 4.0 & 85 & 109 & 1.6 & 104 & 120 & 0.3 & 97 & 111 & 6.4 & 130 & 119 & 5.3 & 114 \\
\hline 81 & Pyraclofos & 108 & 1.7 & 141 & 107 & 1.4 & 157 & 191 & 5.5 & 161 & 104 & 0.3 & 164 & 109 & 3.8 & 151 & 108 & 5.4 & 185 & 108 & 3.4 & 199 \\
\hline 82 & Pyridaben & 120 & 1.1 & 89 & 121 & 1.4 & 96 & 83 & 1.6 & 103 & 118 & 2.0 & 116 & 118 & 2.5 & 117 & 118 & 1.9 & 113 & 117 & 5.4 & 130 \\
\hline 83 & Pyrifenox & 97 & 0.9 & 79 & 94 & 2.3 & 81 & 102 & 2.7 & 85 & 98 & 3.1 & 119 & 85 & 0.6 & 100 & 99 & 7.1 & 138 & 117 & 2.0 & 130 \\
\hline 84 & Pyrimidufen & 82 & 1.3 & 94 & 85 & 2.8 & 101 & 86 & 7.0 & 107 & 114 & 2.3 & 120 & 111 & 3.3 & 125 & 113 & 3.2 & 138 & 120 & 2.2 & 132 \\
\hline 85 & Pyriproxyfen & 99 & 0.8 & 87 & 103 & 1.2 & 89 & 106 & 2.2 & 93 & 109 & 1.1 & 119 & 90 & 3.2 & 117 & 110 & 5.4 & 120 & 116 & 0.9 & 130 \\
\hline 86 & Quinalphos & 98 & 0.7 & 80 & 98 & 2.1 & 84 & 104 & 2.0 & 89 & 118 & 1.7 & 114 & 111 & 3.3 & 93 & 102 & 6.8 & 117 & 115 & 7.0 & 102 \\
\hline 87 & Silafluofen & 88 & 0.1 & 90 & 119 & 1.1 & 92 & 118 & 1.9 & 92 & 176 & 2.6 & 119 & 137 & 2.1 & 115 & 143 & 2.9 & 120 & 147 & 1.5 & 128 \\
\hline 88 & Tebuconazole & 109 & 2.7 & 82 & 102 & 0.5 & 84 & 117 & 3.0 & 93 & 135 & 4.2 & 132 & 112 & 1.8 & 111 & 106 & 4.5 & 133 & 113 & 7.8 & 136 \\
\hline 89 & Tebufenpyrad & 112 & 1.8 & 89 & 108 & 2.2 & 91 & 119 & 2.1 & 92 & 114 & 2.7 & 114 & 114 & 1.0 & 111 & 115 & 3.4 & 129 & 118 & 2.1 & 130 \\
\hline 90 & Tefluthrin & 118 & 1.8 & 76 & 116 & 2.9 & 79 & 117 & 3.1 & 80 & 186 & 2.9 & 86 & 207 & 2.2 & 78 & 168 & 4.0 & 114 & 190 & 3.0 & 79 \\
\hline 91 & Terbufos & 120 & 0.9 & 75 & 122 & 2.4 & 78 & 120 & 1.3 & 81 & 156 & 0.5 & 111 & 109 & 1.3 & 101 & 116 & 1.8 & 129 & 112 & 1.2 & 108 \\
\hline 92 & Thenylchlor & 100 & 0.4 & 88 & 101 & 1.7 & 85 & 109 & 3.5 & 98 & 110 & 2.5 & 117 & 112 & 1.6 & 95 & 113 & 5.4 & 119 & 115 & 6.1 & 129 \\
\hline 93 & Thiobencarb & 96 & 0.6 & 77 & 97 & 1.1 & 78 & 101 & 2.3 & 81 & 118 & 1.9 & 95 & 111 & 1.9 & 75 & 115 & 1.3 & 105 & 116 & 1.2 & 86 \\
\hline 94 & Thiometon & 103 & 1.2 & 74 & 107 & 1.8 & 75 & 107 & 2.6 & 79 & 150 & 2.8 & 93 & 100 & 3.1 & 84 & 104 & 2.5 & 120 & 110 & 4.1 & 88 \\
\hline 95 & Tolclofos-methyl & 97 & 0.9 & 76 & 97 & 1.5 & 80 & 101 & 2.5 & 82 & 117 & 1.8 & 91 & 106 & 2.2 & 84 & 118 & 1.6 & 118 & 106 & 2.9 & 88 \\
\hline 96 & Triadimenol & 102 & 1.4 & 79 & 97 & 3.5 & 82 & 109 & 5.1 & 91 & 110 & 2.2 & 86 & 112 & 3.7 & 114 & 112 & 7.8 & 116 & 117 & 2.1 & 117 \\
\hline 97 & Tricyclazole & 94 & 3.2 & 94 & 100 & 2.4 & 100 & 104 & 4.6 & 111 & 105 & 5.4 & 110 & 111 & 1.9 & 84 & 101 & 7.0 & 92 & 109 & 7.5 & 108 \\
\hline
\end{tabular}




\subsection{Calibration curves for the standard mixture}

To assess the method as a quantitative tool, three standard solutions of all the pesticides $(50,100$, and $500 \mathrm{ng} / \mathrm{mL}$ ) were determined by GPC-GC/MS. Each calibration point was obtained based on three duplicate injections of standard sample. In our experience, some pesticides are weakly absorbed to the inner surface of the capillary column. To adequately fit the calibration curve for all pesticides, a second-order calibration curve was employed. The curve between the peak area $(y)$ and the concentration $(x, \mathrm{ng} / \mathrm{ml})$ was then investigated. The regression equations for representative pesticides, including organophosphorus, organochlorine, organonitrogen, carbamate, and thiocarbamate substances are provided in Table 2 .

\subsection{GPC-GC/MS analysis of pesticides in agricultural products}

Ninety-seven target pesticides were spiked at a concentration level of $0.1 \mathrm{mg} / \mathrm{kg}$ into several kinds of agricultural products, including potato, cabbage, carrot, apple, orange, cucumber, and rice. Total ion chromatograms (TIC) of the spiked potato sample (adding standard pesticides $0.1 \mathrm{mg} / \mathrm{kg}$ ) and the unspiked potato sample are shown in Fig. 3.

Table 3 provides estimated limits of detection (LOD) and quantitation (LOQ), calculated as the concentration that produced a signal that was 3-times and 10-times the background noise level, respectively, for three analyzed matrices: potato, apple, and rice.

Recovery of the pesticides $(0.1 \mathrm{mg} / \mathrm{kg})$ spiked into potato, cabbage, carrot, apple, orange, cucumber, and rice was investigated via GPC-GC/MS. To calculate the recovery, the spiked sample from each agricultural product was prepared three times and the unspiked samples were also investigated. Average recovery results (background of unspiked samples were deducted from the spiked recovery) and the relative standard deviation (R.S.D.) are listed in Table 4. Most pesticides were recovered within an acceptable recovery range from 70 to $120 \%$. Several exceptional recoveries $(>150 \%)$ probably arose from interferences remaining in the matrix (e.g., the conjugation effect of some endogenous compounds may contribute to high recoveries), whereas some pesticides, such as acephate, captan, dichlofluanid, and methamidophos, showed low recoveries $(<70 \%)$ in some matrices. As acephate and methamidophos are highly water-soluble pesticides, they would probably move into the water layer during the extraction step. Other unstable compounds such as captan and dichlofluanid may have been decomposed during the extraction step or the analysis process. More pesticides in vegetables and fruits showed acceptable recoveries than those in rice because of the more complicated matrix of rice. It is apparent from Table 4 that R.S.D. values were generally $<10 \%$. The low R.S.D. values indicate the high reproducibility of analyses of this newly developed GPC-GC/MS system.

\subsection{Comparison of the GPC-GC/MS and GC/MS systems}

The recovery test was also investigated using a conventional GC/MS system. The results are shown in Table 4. Comparing the average recovery data, it is easy to find much better recovery results with GPC-GC/MS than with GC/MS method. For example, in GPC-GC/MS, 83 of 97 pesticides showed acceptable recovery for orange, but in GC/MS, only 54 pesticides showed acceptable recovery. The inherent characteristics of GPC have proved highly advantageous in sample pre-treatment to minimize matrix interferences associated with limited solvent extraction protocols.

\section{Conclusion}

An automated GPC-GC/MS analysis system for the determination of multiple pesticides was developed. Recovery results in the presented pesticides analysis demonstrated that this newly developed system is superior to the conventional GC/MS. The method is accurate and rapid to measure a diverse range of pesticides in agricultural products and it is possible to be of use as a routine tool in monitoring pesticide residues.

\section{Acknowledgements}

The authors gratefully acknowledge financial support from the National Natural Science Foundation of China (Nos. 20437020, 20575073) and the Major Research Program of Chinese Academy of Sciences (KZCX3-SW-432).

\section{References}

[1] C.G. Zambonin, M. Quinto, N.D. Vietro, F. Palmisano, Food Chem. 86 (2004) 269.

[2] B. Albero, C.S. Brunete, J.L. Tadeo, Talanta 66 (2005) 917.

[3] F.E. Ahmed, Trends Anal. Chem. 20 (2001) 649.

[4] K. Saito, A. Sjödin, C.D. Sandau, M.D. Davis, H. Nakazawa, Y. Matsuki, D.G. Patterson Jr., Chemosphere 57 (2004) 373.

[5] W.W. Zhu, J.R. Allaway, J. Chromatogr. A 1055 (2004) 191.

[6] M.I. Venkatesan, T. Northrup, C.R. Phillips, J. Chromatogr. A 942 (2002) 223.

[7] G.G. Rimkus, M. Rummler, I. Nausch, J. Chromatogr. A 737 (1996) 9.

[8] J.J. Vreuls, R.J.J. Swen, V.P. Goudriaan, M.A.T. Kerkhoff, G.A. Jongenotter, U.A.T. Brinkman, J. Chromatogr. A 750 (1996) 275.

[9] A. Pauwels, A. David, P. Schepens, P. Sandra, Int. J. Environ. Anal. Chem. 73 (1999) 171.

[10] Y. Hashi, H.X. Zhou, Y.P. Qin, Chin. J. Anal. Sci. 21 (2005) 441.

[11] M. Anastassiades, S.J. Lehotay, D. Stajnbaher, F.J. Schenck, J. AOAC Int. 86 (2003) 412.

[12] H. Obana, K. Akutsu, M. Okihashi, S. Hori, Analyst 126 (2001) 1529.

[13] S. Butz, T. Heberer, H.J. Stan, J. Chromatogr. A 677 (1994) 63.

[14] S. Butz, H.J. Stan, J. Chromatogr. 643 (1993) 227.

[15] V. Coquart, M.C. Hennion, Sci. Total Environ. 132 (1993) 349.

[16] T. Cserhati, E. Forgacs, J. Chromatogr. 643 (1993) 331.

[17] J. Nolte, H. Mayer, M.A. Khalifa, M. Linscheid, Sci. Total Environ. 132 (1993) 141

[18] S. Chiron, E. Martinez, D. Barcelo, J. Chromatogr. A 665 (1994) 283.

[19] A.D. Corcia, M. Marchetti, Anal. Chem. 63 (1991) 580. 